\title{
An Automated Course-SPECific Vocabulary Identification Program
}

\author{
Variawa, C., and McCahan, S. \\ Department of Mechanical and Industrial Engineering, University of Toronto, Ontario Canada \\ variawa@mie.utoronto.ca; mccahan@mie.utoronto.ca
}

\section{INTRODUCTION}

Professional engineering language is often used to precisely describe specific objects, processes, or situations but may not be taught to students in the engineering classroom as an explicit course objective. Though the method of teaching this vocabulary varies, students' mastery in understanding this corpus is usually assessed (explicitly or implicitly) using written tests and exams based on course content.

As instructors develop their courses around specific learning outcomes, it can become difficult to accurately characterize the vocabulary that ought to be learned. In particular, the words may change over time and with instructor, the ability to discern course-specific from non-course-specific words can be subjective, and the word list of required vocabulary may not be feasible to produce manually. In addition, if the list of vocabulary is not defined a priori, then the transparency of the learning outcomes and assessment validity are reduced.

One approach to developing a critical vocabulary list is to deploy an automated strategy that statistically produces a list of "course-specific" words with minimal user intervention. The strategy relies on analyzing recent existing teaching materials for characteristic vocabulary. This dynamic approach maximizes objectivity while giving the instructor a starting point for defining a list of necessary vocabulary.

The general field of language analysis using automated computational approaches falls under a category of computer science and engineering defined as artificial intelligence. Specifically, words are being translated from human vocabulary to statistical values, and then combined to form a hierarchy of diagnostic keywords for a given document. The output of such an approach would be a word list of coursespecific terms.

For this study, the researchers modified an algorithm called Term Frequency-Inverse Document Frequency (TF-IDF) to classify the vocabulary on a group of engineering exams. This algorithm first calculates the term frequency of each word on an exam by dividing the number of occurrences of a word by the total number of words in that document. The term frequency is then multiplied by the inverse document frequency, which is the logarithm of the number of documents in a comparator set divided by the number of documents in this set containing the word. This factor is dependent on the sample size; so, the more documents that are available in the database, the more accurate the output score for the word. Then, the word and its TF-IDF score are tabulated.

This method appears to show a correlation between course-specific words and TF-IDF score, but some common words also have high TF-IDF scores. To mitigate this, the researchers develop another word list using an identical TFIDF approach, but this time using a different comparator set. The first word list is created by comparing an exam to all CEEA13; Paper 59.

Montreal, QC; June 17-20, 2013 exams in the same discipline. The second word list is created by comparing the same exam to exams in all of engineering. The first score is subtracted from the second resulting in a word list that differentiates course-specific vocabulary more reliably.

\section{RESULTS/DISCUSSION}

The automated method is used at the University of Toronto (UofT) on a database of 2251 engineering exams from the years 2007-2011. The method was coded into a software program that automates serial tasks such as optical character recognition, content organization, and processing of calculated results and words.

The authors combined several instances of one course CHE230 "Environmental Chemistry" - over a span of three years, 2009-2011, into a large word list. This wordlist is then compared to the 21 million word UofT Engineering Corpus using the TF-IDF algorithm. The resulting wordlist is graphically presented in Fig 1.

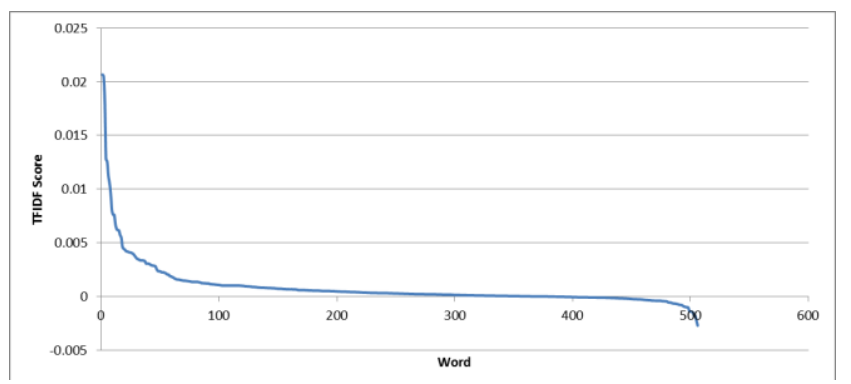

Figure 1 - Shows the combined results of CHE230 over a 3 year period. The TFIDF score is an indicator of keywords, and appears to plateau around the 75-word mark

The figure above shows that the first 75 or so words on this list have a significantly higher modified TF-IDF score than the rest of the 507 words. Since the score is a measure of how characteristic a word is to a given document (in this case, 3 instances of the same exam), the first 75-words can be investigated in detail to see whether these appear to be course or domain-specific words. The long "plateau" of words, starting around 75 on the x-axis, are those that appear just as frequently in the CHE230 exams as they do in all of engineering, while the tail-end around 500 are words that occur less frequently in CHE230, than in engineering in general.

Theoretically, high scoring words should be the only ones that are characteristic to the document being investigated. To test whether the vocabulary is properly sorted, the authors are working with the course staff of CHE230 and a linguistics expert to identify whether the method has captured an appropriate word list. Future work includes increasing the robustness of this method by sifting words that are misspelled, or are non-English words. The goal is to reduce inaccessible language while promoting technical vocabulary development. 